\title{
Characteristic Analysis of Electromagnetic Multi-Shift of Interior Permanent Magnet Synchronous Motor
}

\author{
Hong-Sik Lim, Do-Hyun Kim, Ki-Chan Kim* \\ Department of Electrical Engineering, Hanbat National University, South Korea
}

Copyright $(2019$ by authors, all rights reserved. Authors agree that this article remains permanently open access under the terms of the Creative Commons Attribution License 4.0 International License

\begin{abstract}
Background/ Objectives: In order to increase the travel distance of an electric vehicle, it is important to widen the efficiency range of the motor. In this paper, we study the electromagnetic multi-shift method of the motor. Methods/ Statistical analysis: We will study the expansion of the efficiency range of the motor by applying three electromagnetic multi-shift methods. In addition, the characteristics, advantages and disadvantages of methods that switch finite element analysis methods (FEMs) are analyzed. Findings: In this paper, we study the characteristic analysis of the electromagnetic multi-shift of interior permanent magnet synchronous motor (IPMSM). For electric vehicles, the Driving Range is important. Therefore, the expansion of the range of maximum efficiency is more important than the improvement of the maximum efficiency point of the motor. Three electromagnetic transmission methods were applied and analyzed to increase the efficiency range. The first method is the high efficiency range appears to be expanded in the high-speed range, but the influence of the expansion of the maximum efficiency range is not significantly expanded as compared with the other method. In addition, second method and third method in the range of maximum efficiency are similar. However, when third method is the maximum efficiency value increases up to about $8,500 \mathrm{rpm}$ operation range at about 5,000 rpm. Improvements/Applications: The efficiency of the motor to which various electromagnetic multi-shift methods were applied was derived by FEM and the characteristics were analyzed.
\end{abstract}

Keywords Electromagnetic Multi-shift, IPMSM, Efficiency Map, Number of Serial Turns per Phase, Switching Y $\Delta$, Number of Parallel Circuits

\section{Introduction}

Due to energy problems and exhaustion problems of fossil fuels worldwide, we are interested in the development of environment-friendly electrical devices and efficient electrical devices. In particular, development of environmentally friendly vehicles and research on alternative energy tend to be in the limelight, such as electric vehicles (EVs) [1]-[4]. An internal combustion engine driven by an engine uses a mechanical transmission. However, the mechanical transmission occupies a lot of space of the car and has problems such as weight and durability. Generally, IPMSM are often used as drive motors for electric vehicles. The IPMSM is a form in which a high performance rare earth permanent magnet of a rotor core is inserted. This provides high mechanical strength at high-speed operation, high power density and high efficiency. In particular, IPMSM use magnetic torque and reluctance torque simultaneously. In addition, the control is advantageous in the field of weak field, which enables wide operation [5]-[7]. The driving curve of the IPMSM for driving the EVs shows the same characteristic as the driving curve of an ideal vehicle through about field weakening control at high-speed driving. However, the efficiency varies with the operating point, and generally occurs at the point of reaching the voltage limit for maximum efficiency. Therefore, the drive range by electric vehicles is not the point of maximum efficiency, but is affected by the range of efficiencies [8]-[10].

In this paper, we study the electromagnetic multi-shift method of the motor. Three methods are applied to increase the efficiency range of the motor. Also, each method is applied, and the motor is analyzed by FEM to analyze the attribute and the advantages and disadvantages [11].

\section{Analysis Model}

FEM analysis model is shown in figure 1. The basic model is an IPMSM in which permanent magnets are inserted in the rotor. As shown in the figure, the permanent magnet of the rotor is designed in the reverse triangle shape and consists of 8 poles and 48 slots. The stack length is 140 $\mathrm{mm}$, and the stator diameter is $200 \mathrm{~mm}$. 

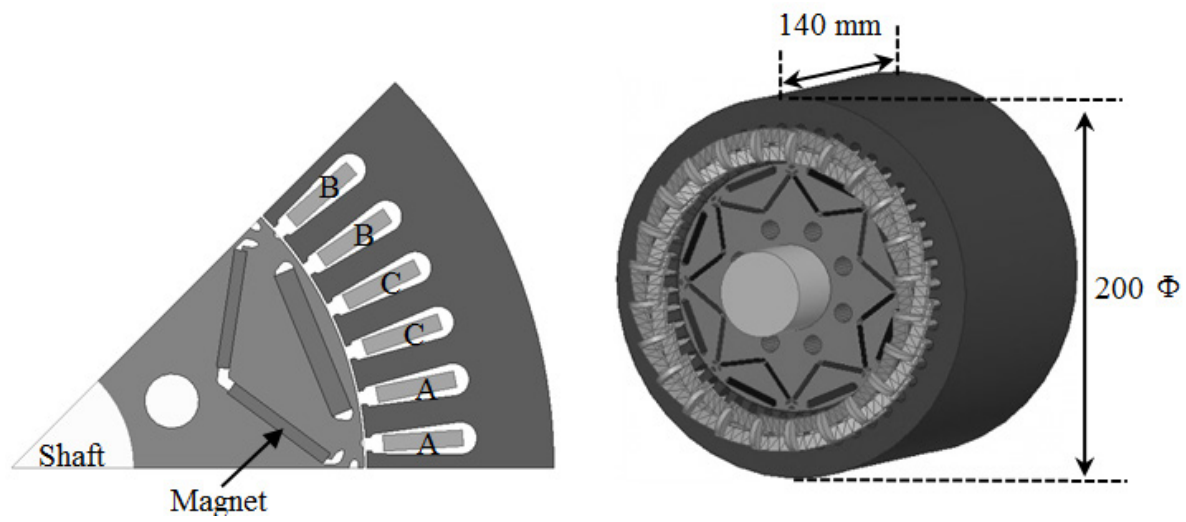

Figure 1. 2D\&3D FEM analysis model (a) 2D FEM model (b) 3D FEM model

The specifications of the analytical model are shown in table 1. As shown in the table, the instantaneous output of the basic model is $80 \mathrm{~kW}$ and the continuous rated output is designed to $40 \mathrm{~kW}$. In addition, the rated operation speed is 3,000 $\mathrm{rpm}$ and the maximum operation speed is $10,000 \mathrm{rpm}$.

Table 1. The specification of analysis model

\begin{tabular}{ccc}
\hline Parameter & Value & Unit \\
\hline Number of Pole / Slot & $8 / 48$ & $\mathrm{~kW}$ \\
\hline Maximum Power & 80 & $\mathrm{Rpm}$ \\
\hline Speed (Base / Max.) & $3,000 / 10,000$ & $\mathrm{~mm}$ \\
\hline Stator (Outer / Inner) & $200 / 131$ & $\mathrm{~mm}$ \\
\hline Rotor (Outer / Inner) & $130 / 45$ & $\mathrm{~mm}$ \\
\hline Stack & 140 & - \\
\hline Parallel branches & 4 & - \\
\hline Num. of Reels per slot & 12 & - \\
\hline Num. of Turns per slot & 9 &
\end{tabular}

Figure 2 shows the TN curve of a basic model of $80 \mathrm{~kW}$. At 3,000 rpm the torque is $254.6 \mathrm{Nm}$ and at $10,000 \mathrm{rpm}$ a torque of $76.39 \mathrm{Nm}$ can be delivered. Figure 2 shows an efficiency map of all operating ranges of the basic model of the interior permanent magnet synchronous motor. As shown in the figure, the highest efficiency can know because the voltage saturation is near the instantaneous rated operating point. The maximum efficiency point is shown at about 5,500 rpm as shown.
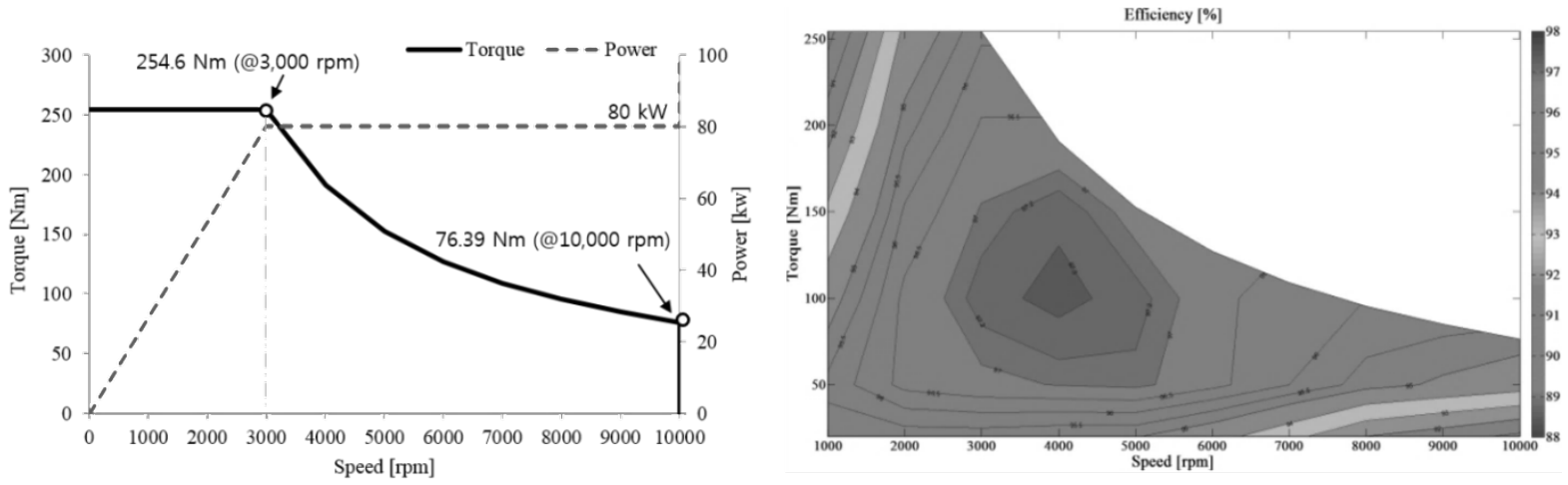

Figure 2. T-N curve and Efficiency Map in Base Model 


\section{IPMSM Characteristics Applying Electromagnetic Multi-shift Method}

\subsection{The Method of Changing the Number of Serial Turns}

The first method is to use a semiconductor switching element to switch the number of turns. This is a method of reducing the number of series turns in a high-speed operating range for driving a motor. Figure 3 shows a circuit diagram in which the equivalent serial turn number is changed. As shown, vector control of IPMSM is performed thought an inverter [12]. DB1 and DB2 indicate three-phase diode bridges. Further, the number of serial turns per phase can be changed the winding changeover switch.

\subsection{The Method of Switching Y- $\Delta$}

Figure 4 shows a $\mathrm{Y}-\Delta$ switching circuit diagram. As shown in Figure 4, the $\mathrm{Y}$ connection switch is turned ON during low-speed operation with Y connection. Further, at High-speed operation, the $\mathrm{Y}$ connection switch is turned off and the delta connection switch is turned on. Although high torque can be output during low-speed operation, the saturation point of voltage becomes low at low-speed and the range of maximum efficiency is displayed at low-speed. In addition, although switching to the delta wire connection is performed the changeover switch of the winding part, low torque during operation is generated. However, a voltage limit margin occurs and the voltage saturation point is delayed. Therefore, there is an advantage that high-speed and wide area efficiency range can be expanded [13]-[14].

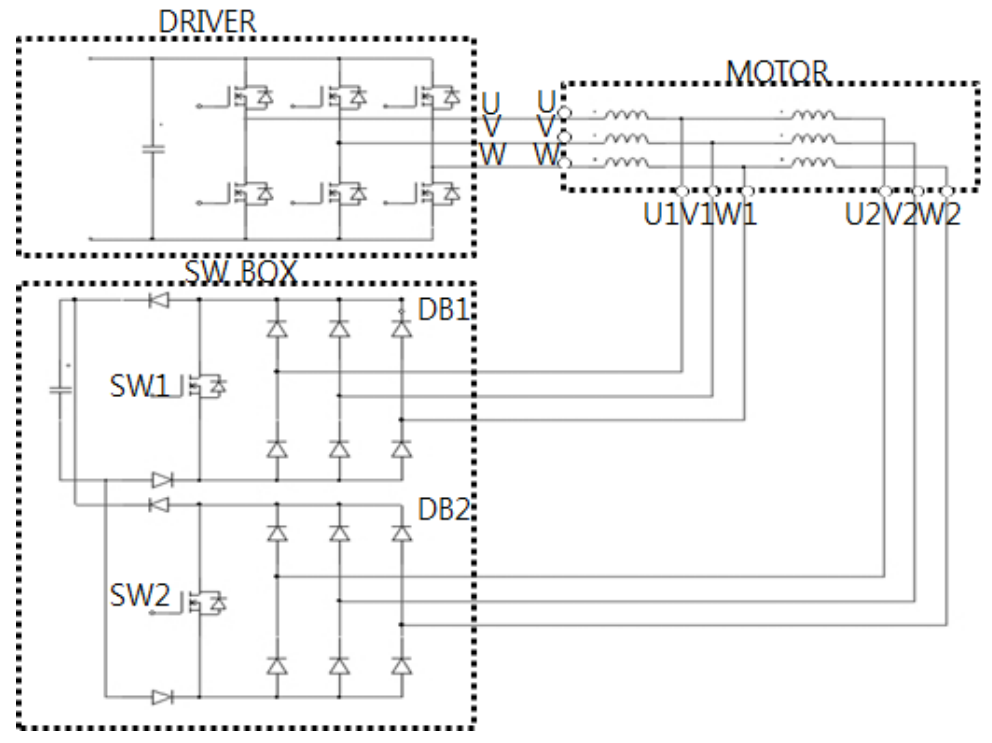

Figure 3. Number of serial turn per phase circuit diagram

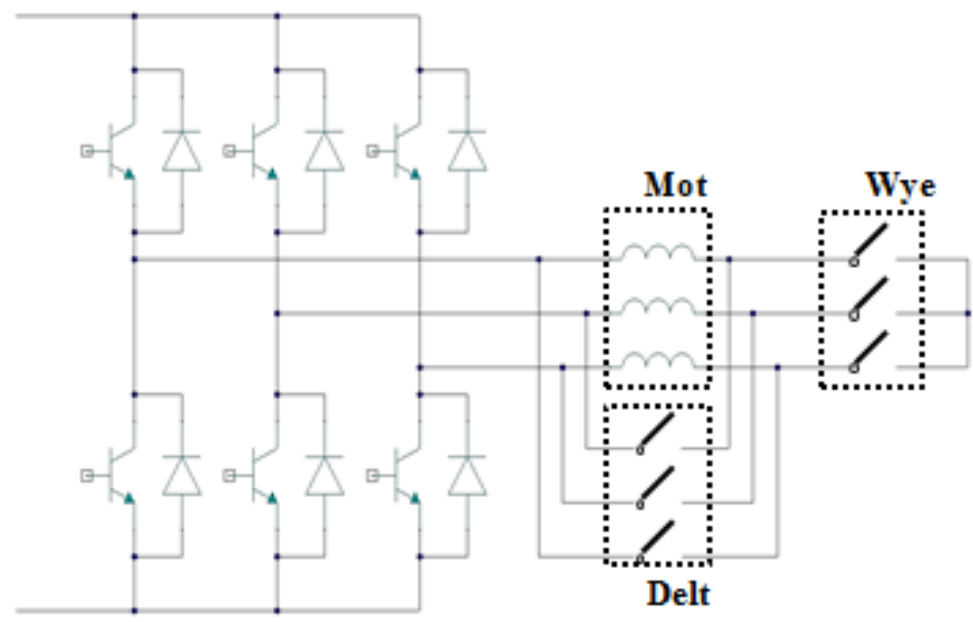

Figure 4. $\mathrm{Y}-\Delta$ connection circuit diagram 


\subsection{The Method of Changing the Number of Parallel Circuits}

The third method is a method of changing the number of parallel circuits. Unlike the first method, it has the feature of using all windings not using unnecessary parts. Figure 5 shows a circuit diagram of the third method. The number of parallel circuits is two as shown in Figure 5. The switches of the circuit use thyristors. The thyristor controls the current and is driven as shown in figure 5. In addition, As shown in figure 5 , two parallel operation is performed during low-speed driving, and four parallel operation is performed during high-speed operation. high torque can be output, and the viewpoint of voltage saturation is accelerated at low-speed, and the range of maximum efficiency is displayed at low-speed. In addition, at the time of high-speed operation, there is a voltage limit margin, high-speed, wide area operation is possible, and the range of maximum efficiency is expanded.

\subsection{Characteristic Map Comparison Applied Electromagnetic Multi-shift Method}

Figure 6 shows an efficiency map to which the electromagnetic multi-shift method is applied in the complementary design model. As shown in the figure, when applying a total of three methods, the range of maximum efficiency was expanded compared to before applying the electromagnetic multi-stage gear shifting method.

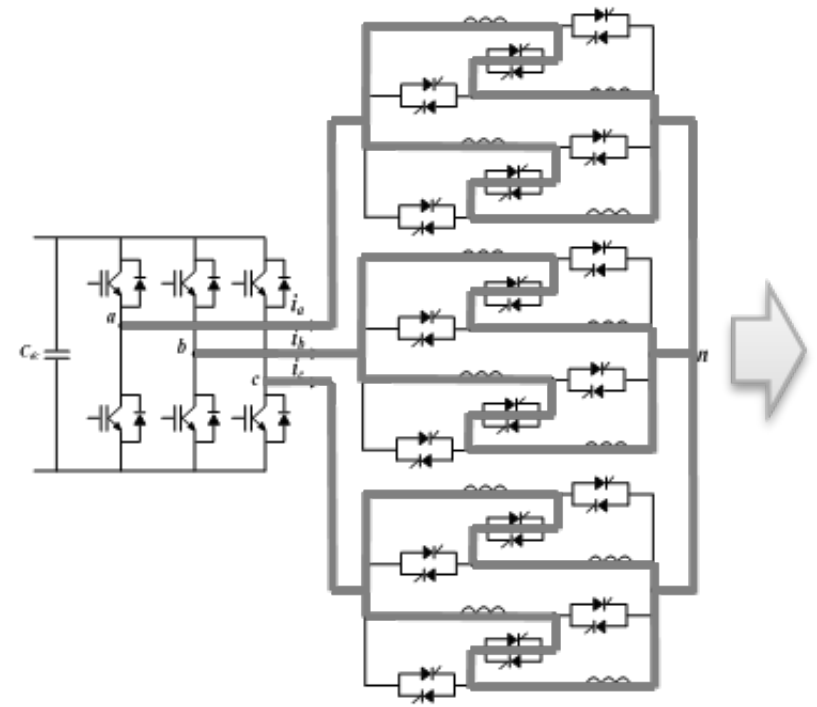

(a) number of parallel circuits: 2

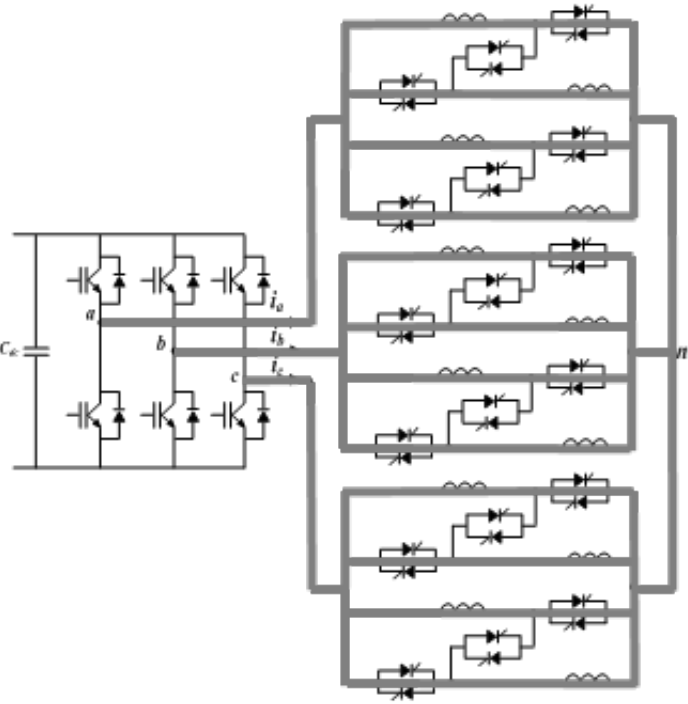

(b) number of parallel circuits: 4

Figure 5. Changing the number of parallel circuits diagram

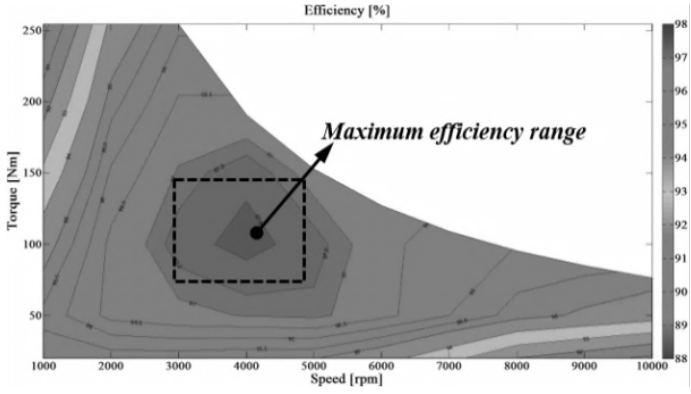

(a) base motor

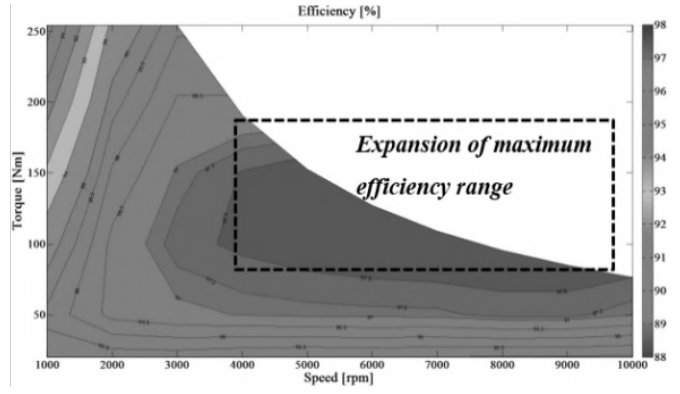

(b) number of serial turn per phase 


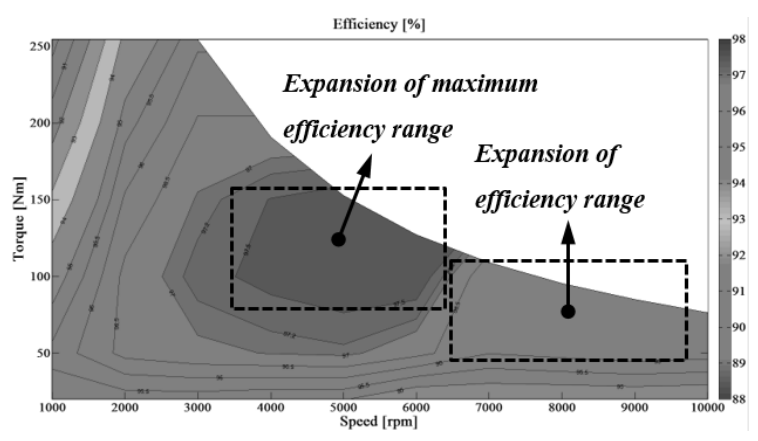

(c) Y- $\Delta$ connection method

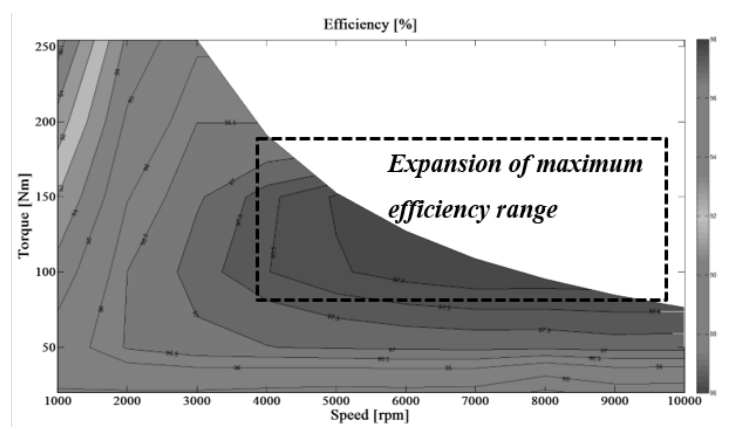

(d) number of parallel circuits method

Figure 6. Efficiency map for each method

\section{Conclusions}

In this paper, we study the characteristic analyzed of electromagnetic multi-shift method of IPMSM. For electric vehicles, the driving range are important. Therefore, the extension of the range of maximum efficiency is more important than the improvement of the maximum efficiency point of the motor. so as to extend the range of maximum efficiency, a total of three electromagnetic multistage shifting methods were applied. The first method has an advantage that the number of serial turns per phase is reduced at high-speed, thereby reducing copper loss and expanding the maximum efficiency range to a high-speed region. However, the range of maximum efficiency is smaller than the other methods. From the point of view of motor design, there is a disadvantage that the operation with a reduced number of serial turns considerably in the high-speed operation range occupies a necessary part in terms of material cost. The second method not only secures a wide operation zone, but also can reduce the use of current in the field region and secure a control margin. In addition, the maximum efficiency range when switching from low-speed operation to high-speed operation has been greatly expanded. The third method is characterized in that the resistance of the winding is reduced in the high-speed operation zone, the copper loss is reduced, and the efficiency is improved. However, many thyristors for current control are used to change the number of parallel circuits. It therefore has the disadvantages of weight and size of the thyristor.

\section{Acknowledgements}

This work was supported by the Korea Institute of Energy Technology Evaluation and Planning(KETEP) and the Ministry of Trade, Industry \& Energy(MOTIE) of the Republic of Korea (NO.20184030201900).

\section{REFERENCES}

[1] Won-Ho Kim, Mi-Jung Kim, Ki-Doek Lee, Jae-Jun Lee, Jung-Ho Han, Tae-Chul Jeong, Su-Yeon Cho, and Ju Lee, "NE-Map-Based Design of an IPMSM for Traction in an EV", IEEE Transactions on Magnetics, Vol. 50, No. 1, Art. No. 4001404, Jan. 2014.

[2] H. W. Lee, K. D. Lee, W. H. Kim, I. S. Jang, M. J. Kim, J. J. Lee, J. Lee, Parameter design of IPMSM with concentrated winding considering partial magnetic saturation, IEEE Transactions on Magnetics, 2011, 10 (10), pp. 3653-3656.

[3] Liang Chen, David Hopkinson, Jiabin Wang, Andrew Cockburn, Martin Sparkes, William O'Neill, Reduced Dysprosium Permanent Magnets and Their Applications in Electric Vehicle Traction Motors, IEEE Transactions on Magnetics, 2015, 51 (11).

[4] Thanh Anh Huynh and Min-Fu Hsieh, "Comparative Study of PM-Assisted SynRM and IPMSM on Constant Power Speed Range for EV Applications", IEEE Transactions on Magnetics, Vol. 53, No. 11, Art. No. 8211006, Nov. 2017.

[5] Dong-Jun Kim, Do-Kwan Hong, Jae-Hak Choi, You-Do Chan, Byung-Chul Woo, and Dae-Hyun Koo, "An Analytical Approach for a High-speed and High Efficiency Induction Motor Considering Magnetic and Mechanical Problems", IEEE Transactions on Magnetics, Vol. 49, No. 5, pp. 2319-2322, MAY. 2013.

[6] Elsayed, B. A., Elhenawy, A. A., \& Sultanah, A. S. Synthesis, Characterization, Antimicrobial and Cytotoxic Studies on Some Novel Transition Metal Complexes of Schiff Base Ligand Derived From Sulfadiazine with Molecular Orbital Calculations. International Journal of Chemistry and Materials Research, 2014, 2(1), pp.1-16.

[7] El-Shorbagy, M., \& Mousa, A. Chaotic particle swarm optimization for imprecise combined economic and emission dispatch problem. Review of Information Engineering and Applications, 2017, 4(1), pp. 20-35.

[8] Enyenihi, H. J., Rosemary, N. C., \& Eduediuyai, D. Development of Web Application for University of Uyo Post UTME Examination Timetable. Review of Computer Engineering Research, 2018, 5(2), pp.64-73. 
[9] Hajirahimova, M., \& Aybeniz, A. Current Approaches in Prediction of PVT Properties of Reservoir Oils. Review of Information Engineering and Applications, 2018,5(2), pp.31-40.

[10] Min-Fu Hsieh, Feng-Sheng Hsu, David G. Dorrell, "Winding Changeover Permanent-Magnet Generators for Renwable Energy Applications", IEEE Transactions on Magnetics, Vol. 48, No. 11, 4168-4171, Nov. 2012.

[11] Hyung-Woo Lee, Ki-Dock Lee, Won-Ho Kim, IK-Sang Jang, Mi-Jung Kim, Jae-Jun Lee, and Ju Lee, "Parameter Design of IPMSM With Concentrated Winding Considering Partial Magnetic Saturation", IEEE Transactions on Magnetics, Vol. 47, No. 10, pp. 3653-3656, Oct. 2011.

[12] Jian Li, Dawoon Choi, Yunhyun Cho, "Analysis of Rotor Eccentricity in Switched Reluctance Motor With Parallel Winding Using FEM", IEEE TRANSACTIONS ON MAGNETICS, VOl. 45, NO. 6, pp. 2851 2854, Jun. 2009.

[13] Sang-Hyeok Seo, Gyeong-Jae Park, ByungKwan Son, Yong-Jae Kim and Sang-Yong Jung, "Novel Design Method to Reduce Input Current for Multi-Operating Point IPMSM", IEEE Transactions on Magnetics, Vol. 53, No. 6, Art. No. 8104804, Jun. 2017.

[14] Byeong-Hwa Lee, Kyu-Seob Kim, Jae-Woo Jung, Jung-Pyo Hong, Young-Kyoun, Temperature Estimation of IPMSM Using Thermal Equivalent Circuit, IEEE Transactions on Magnetics, 2012, 48 (11), pp. 2949-2952. 